\title{
Transplante cardíaco na displasia arritmogênica do ventrículo direito*
}

\author{
Heart transplantation in the arrhythmogenic \\ right ventricular dysplasia
}

\author{
Clarissa Nóbrega Gambarra Nascimento', Lucas Burim Vilas \\ Boas ${ }^{1}$, Carlo Felício Campagnoli Napolitano², Guilherme \\ Henrique Bianchi Coelho ${ }^{3}$, Ronaldo Honorato ${ }^{4}$, \\ Alfredo Inácio Fiorelli ${ }^{5}$
}

\begin{abstract}
Nascimento CNG, Vilas Boas LB, Napolitano CFC, Coelho GHB, Honorato R, Fiorelli Al. Transplante cardíaco na displasia arritmogênica do ventrículo direito. Rev Med (São Paulo). 2009 jan.-mar.;88(1):36-44.

RESUMO: Introdução: A displasia arritmogênica do ventrículo direito (DAVD) é uma doença do miocárdio de origem familiar, caracterizada pela substituição do miocárdio por tecido fibrogorduroso, predominantemente no ventrículo direito, levando a focos de arrritmia, podendo levar à morte súbita. Objetivo: O presente trabalho tem por objetivo apresentar a evolução clínica de quatro pacientes portadores de DAVD que foram submetidos ao transplante cardíaco ortotópico pela técnica bicaval. Método e Casuística: Foram levantados os prontuários de quatro pacientes portadores de DAVD, submetidos ao transplante cardíaco, que evidenciou em todos os casos evolução rápida e grave com insuficiência cardíaca classe funcional(NYHA) III/ IV, arritmias de difícil controle e disfunção biventricular, sendo, portanto, indicado o transplante. Resultados: Em todos os casos o transplante foi realizado pela técnica bicaval com anuloplastia profilática da valva tricúspide. Apenas um paciente evoluiu com rejeição hiperaguda e infecção, indo a óbito no $7^{\circ}$ dia de pós-operatório. Nos outros três casos houve remissão completa dos sintomas. O exame anatomopatológico dos corações explantados confirmou a presença da doença. Conclusões: A DAVD pode ter evolução grave e, nesses casos, o transplante cardíaco deve ser considerado, e mostra-se efetivo, com remissão completa da doença.
\end{abstract}

DESCRITORES: Displasia arritmogênica ventricular direita. Transplante de coração. Insuficiência cardíaca.

\footnotetext{
* Trabalho premiado no COMU - 2008. Área Relato de Caso - Prêmio Oswaldo Cruz, 2008.

1. Acadêmicos do $4^{\circ}$ ano do curso de Medicina da Faculdade de Medicina da Universidade de São Paulo.

2. Acadêmico do $5^{\circ}$ ano do curso de Medicina da Faculdade de Medicina da Universidade de São Paulo.

3. Médico estagiário do Instituto do Coração da Universidade de São Paulo.

4. Médico Assistente, Coordenador do Transplante Cardíaco do Instituto do Coração da Universidade de São Paulo.

5. Médico Assistente, Chefe do Transplante Cardíaco do Instituto do Coração da Universidade de São Paulo.

Endereço para correspondência: Clarissa Nóbrega Gambarra Nascimento. Rua Santa Madalena, 239, Apart. 52, Bela Vista. São Paulo, SP. E-mail: clangn@ hotmail.com
} 
INTRODUÇÃO

A Displasia Arritmogênica do Ventrículo Direito é uma miocardiopatia de caráter degenerativo em que ocorre substituição do miocárdio por tecido fibro-gorduroso principalmente em uma região denominada de triângulo da displasia, delimitado pelo infundíbulo anterior, o ápice do ventrículo direito e o ponto de encontro da face diafragmática com o anel fibroso da valva tricúspide ${ }^{1,15}$ (Figura 1). Esse infiltrado se interpõe às células das fibras de purkinje, gerando um atraso da condução do impulso elétrico. Este fato gera focos ectópicos de estímulos elétricos responsáveis pelas arritmias.

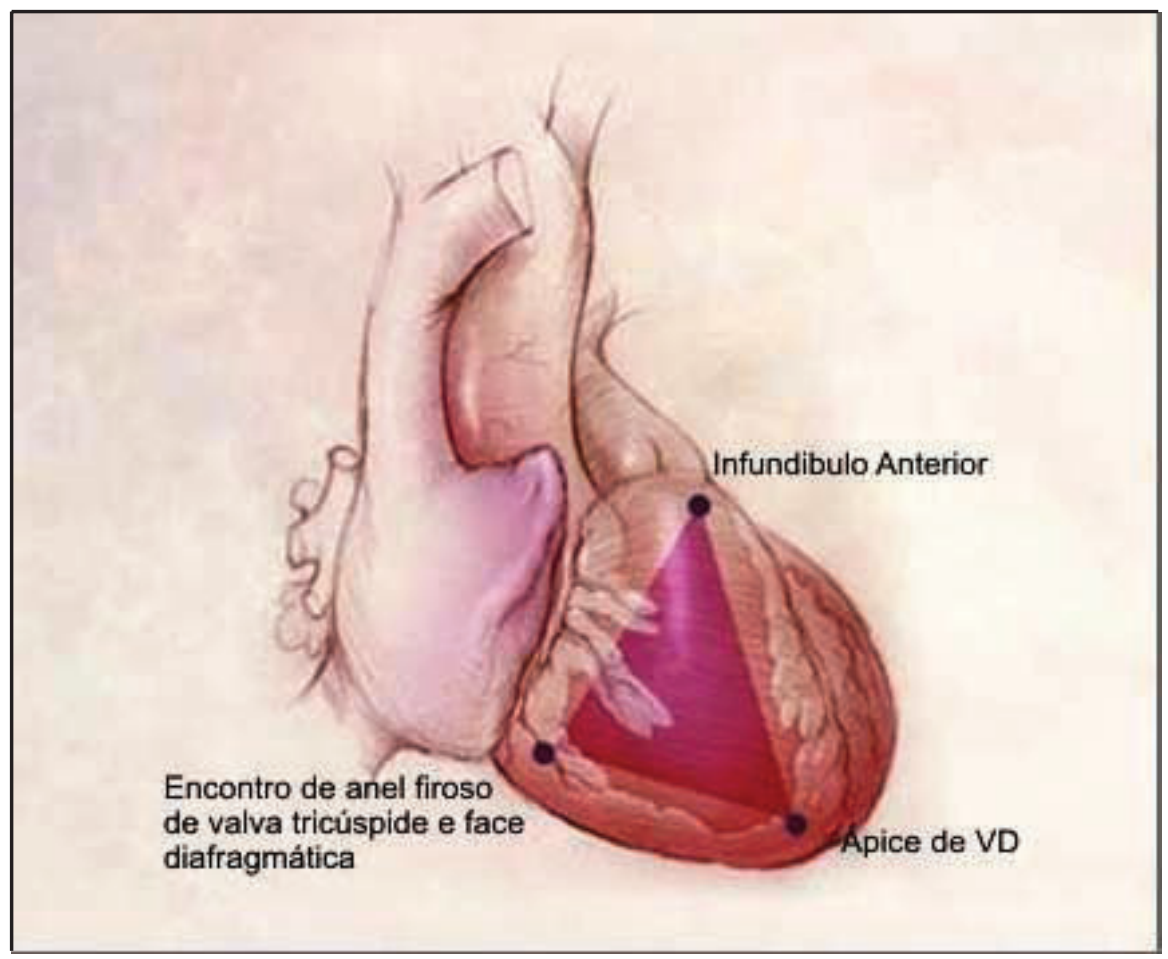

Figura 1. Triângulo da displasia

Ainda, com o avanço dos estudos com genética notou-se que é uma patologia de caráter familiar em $50-80 \%{ }^{12}$ dos casos com padrão de herança autossômica dominante, e penetrância incompleta e variável. Já foram isolados alguns genes responsáveis pelo aparecimento da doença.

A disfunção do ventrículo direito é precoce à do esquerdo e, na sua maioria, é o determinante dos sinais e sintomas, sendo rara a evolução para insuficiência cardíaca, com comprometimento hemodinâmico significativo. O emprego de desfibriladores implantáveis tem sido uma opção para a prevenção de morte súbita por DAVD, no entanto, o aumento da longevidade desses pacientes, que essa terapêutica proporcionou, tem sido um fator importante na evolução para disfunção biventricular ${ }^{13}$ por progressão da substituição fibrogordurosa em septo e ventrículo esquerdo. O transplante cardíaco tem sido uma terapêutica utilizada para pacientes com arritmias malignas graves ${ }^{14,20}$, intratáveis com uso de antiarrítmicos, e com disfunção ventricular comprometendo do débito cardíaco ${ }^{20}$.

\section{OBJETIVO}

O objetivo desse artigo é apresentar a casuística do nosso serviço, de quatro pacientes portadores de DAVD submetidos ao transplante cardíaco.

\section{MÉTODOS}

Foram levantados os prontuários de quatro pacientes submetidos ao transplante cardíaco com DAVD. O diagnóstico clínico foi complementado com o estudo anatomopatológico dos corações explantados no momento do transplante cardíaco, por análise macroscópica e microscópica.

\section{CASUÍSTICA}

Foram realizados 370 transplantes cardíacos no período de 1985 a 2008 , dentre os quais, quatro pacientes $(1,08 \%)$ tinham o diagnóstico de DAVD. A 
Nascimento CNG et al. Transplante cardíaco na displasia arritmogênica do ventrículo direito.

média de idade desses quatro pacientes em anos é $18,7 \pm 2,5(15-21)$ e média de IMC $20,5 \mathrm{~kg} / \mathrm{m}^{2} \pm 1,8$. Os sintomas iniciais apresentados foram: síncope e dispneia progressiva em $50 \%$ dos casos, os demais não apresentavam sintomas quando diagnosticados. Todos evoluíram para ICC classe funcional III-IV e em dois casos com sinais de insuficiência grave de ventrículo direito, com anasarca. Todos os pacientes apresentavam arritmias, sendo necessário o implante de cardiodesfibrilador implantável (CDI) em três casos. Ao ecocardiograma a fração de ejeção (FE) do ventrículo esquerdo variou entre $20 \%$ e $43 \%$ (29,5\% $\pm 11,4)$. Três dos pacientes apresentavam histórico familiar de morte por causa cardíaca, sendo dois com casos de morte súbita com confirmação em necropsia de DAVD.

Tabela 1. Características dos pacientes portadores de DAVD submetidos a transplante cardíaco

\begin{tabular}{lc}
\hline Variável & $2 / 4$ \\
\hline Sexo: masculino & 18,7 anos \\
\hline Idade & 20,5 \\
\hline IMC (índice de massa corpórea em kg/m²) & $3 / 4$ \\
\hline Presença de história familiar & $3 / 4$ \\
\hline Presença de CDI no momento do transplante & $2 / 4$ \\
\hline Sintomáticos no momento do diagnóstico de DAVD & $4 / 4$ \\
\hline Comprometimento biventricular na indicação do transplante & $29,5 \%$ \\
\hline Média de FE de VE & \\
\hline
\end{tabular}

\section{RELATO DOS CASOS}

Caso 1: VDP, sexo feminino, 17 anos, IMC $19 \mathrm{~kg} / \mathrm{m}^{2}$, há cinco anos com história de dispneia progressiva e síncope. Evoluiu para ICC classe funcional III e sinais de insuficiência de ventrículo direito, tendo várias internações em anasarca. Fazia uso contínuo de furosemida, espironolactona, captopril, amiodarona. Ao último ecocardiograma apresentava FE de VE de $35 \%$.

Em junho de 2000 foi submetida ao transplante cardíaco ortotópico bicaval com plastia de valva tricúspide ${ }^{6}$, realizado eletivamente. $O$ doador era do sexo masculino com 22 anos e IMC $22 \mathrm{~kg} / \mathrm{m}^{2}$, cuja causa de morte foi ferimento de arma de fogo, sem uso de noradrenalina e infecção sistêmica na data do transplante.

O tempo de anóxia foi de 72 minutos e de circulação extracorpórea (CEC), 139 minutos. Não apresentou intercorrências intra-operatórias. O esquema imunossupressor inicial era composto por azatioprina, ciclosporina e prednisona.

Caso 2: AGL, sexo feminino, 20 anos, IMC $19,6 \mathrm{~kg} / \mathrm{m}^{2}$, há quatro anos com história de dispneia progressiva e síncope. Evoluiu em ICC classe funcional IV, com comprometimento biventricular e taquicardia ventricular sustentada (TVS) ${ }^{14}$, foi submetida a implante de CDI três anos antes da realização do transplante. Fazia uso contínuo de losartan, espironolactona, furosemida e digoxina. Ao último ecocardiograma apresentava FE de VE de $43 \%$.

Em julho de 2002 foi submetida ao transplante cardíaco ortotópico bicaval com plastia de valva tricúspide $^{6}$, eletivamente. $\mathrm{O}$ doador era do sexo feminino com 43 anos e IMC $20 \mathrm{~kg} / \mathrm{m}^{2}$, cuja causa de morte foi $\mathrm{AVCH}$, com uso de noradrenalina, permanência de cinco dias em UTI e infecção sistêmica na data do transplante.

O tempo de anóxia foi de 86 minutos e de CEC, 120 minutos. Não houve intercorrências intraoperatórias. O esquema imunossupressor inicial foi composto por azatioprina, ciclosporina e prednisona. Permaneceu seis dias em UTI, no pós-operatório.

Caso 3: ESM, sexo masculino, 21 anos, IMC $20,4 \mathrm{~kg} / \mathrm{m}^{2}$, antecedente familiar de morte súbita com menos de 35 anos, e por isso iniciou a investigação diagnóstica. Há quatro anos com história de dispneia progressiva e síncope. Evoluiu para ICC classe funcional IV, com comprometimento biventricular e TVS, sendo submetido a implante CDI três anos antes do transplante. Ao último ecocardiograma apresentava FE de VE de $20 \%$.

Em janeiro de 2006 foi submetido ao transplante cardíaco ortotópico bicaval com plastia de valva tricúspide $^{6}$, eletivamente. $O$ doador era do sexo masculino, 45 anos e IMC $25 \mathrm{~kg} / \mathrm{m}^{2}$, cuja causa de morte foi 
AVCI, com uso de noradrenalina, permanência de dois dias em UTI, sem infecção sistêmica na data do transplante.

O tempo de anóxia foi de 248 minutos e de CEC, 152 minutos. Não apresentou intercorrências intra-operatórias. No pós-operatório imediato evoluiu com resposta inflamatória sistêmica, apresentando disfunção moderada do ventrículo direito, bloqueio átrio-ventricular. Houve piora da função de ventrículo direito associado à disfunção do ventrículo esquerdo no quarto dia, piora atribuída à rejeição hiperaguda, instituído plasmaferese, pulsoterapia com corticoides e mantido antibiótico terapia com cefepime e vancomicina devido ao foco pulmonar infeccioso. O quadro clínico manteve-se grave sendo orientada associação de azitromicina e fluconazol para o foco infeccioso e realizado implante de dispositivo de assistência cardiopulmonar direita no quinto dia de pós-operatório. Ao longo da evolução, houve manutenção do quadro de choque circulatório refratário, falecendo no sétimo dia de pós-operatório.

Caso 4: RIS, sexo masculino, 15 anos, IMC 23 $\mathrm{kg} / \mathrm{m}^{2}$, antecedente familiar de morte súbita aos 35 anos, com laudo de necropsia confirmando presença de DAVD. Iniciou o quadro com febre e sopro cardíaco ao exame físico e dilatação de ventrículo direito, sendo feita a hipótese diagnóstica de miocardite viral ${ }^{2}$. Apresentava também, como antecedente clínico, hipotireoidismo. Evoluiu com dispneia progressiva, chegando a ICC classe funcional IV, com ascite, anasarca sendo internado para compensação. Foi implantado CDI dois anos antes do transplante. Fazia uso contínuo de amiodarona, carvedilol, espironolactona, hidroclortiazida e levotiroxina. Ao último ecocardiograma apresentava FE de VE de $20 \%$.

Em agosto de 2007, foi submetido ao transplante cardíaco ortotópico bicaval com plastia de valva tricúspide ${ }^{6}$, em caráter de prioridade por uso de drogas vasoativas (milrinona e dobutamina). $O$ doador era do sexo masculino com 34 anos e IMC 26 $\mathrm{kg} / \mathrm{m}^{2}$, cuja causa de morte foi traumatismo crânioencefálico, com uso de noradrenalina, permanência de um dia em UTI e sem infecção sistêmica na data do transplante.

O tempo de anóxia foi de 150 minutos e de CEC, 210 minutos. Apresentou parada cardíaca durante a esternotomia, revertida prontamente com massagem cardíaca interna.

Fez uso de prednisona, micofenolato mofetil e ciclosporina como imunossupressão inicial. Ficou nove dias em UTI, durante este período apresentou derrame pericárdico de repetição, sendo necessário reabordagem cirúrgica, e hemorragia subaracnoidea com episódio de convulsão.

As alterações encontradas no eletrocardiograma (ECG) de portadores de DAVD estão elencadas na Tabela 2. O ECG dos pacientes apresentava alterações compatíveis com DAVD, como onda épsilon nas derivações V2 e V3 (Figura 2) e inversão de onde $T$, visualizadas mais facilmente nas derivações V1 e V3.

Tabela 2. Alterações de eletrocardiograma de pacientes portadores de DAVD, derivações onde são mais observadas estas alterações e a porcentagem de pacientes acometidos com tal distúrbio elétrico

\begin{tabular}{llc}
\hline \multicolumn{1}{c}{ Alteração } & \multicolumn{1}{c}{ Derivações } & $\%$ \\
\hline Inversão da onda T & Precordiais direitas & $54 \%$ \\
\hline Duração do complexo QRS $>110 \mathrm{~ms}$ & Precordiais direitas, principalmente em V1* & $30 \%$ \\
\hline Ondas épsilon** & Precordiais direitas & $15 \%$ \\
\hline $\begin{array}{l}\text { Potenciais fragmentados sugestivos de retardo da ativação } \\
\text { ventricular direita }\end{array}$ & $\begin{array}{l}\text { Derivações bipolares em precórdio (derivação } \\
\text { de Lewis e correlatas) }\end{array}$ & $18 \%$ \\
\hline Bloqueio de ramo direito & & Raros \\
\hline Distúrbio de condução pelo ramo direito & & Raros \\
\hline Complexos de baixa voltagem & & $24 \%$ \\
\hline Hipertrofia ventricular direita & & \\
\hline Taquiarritmia supraventricular & & \\
\hline * esta alteração na derivação V1 tem sensibilidade de 55\% e especificidade de 100\%; & \\
** deflexão de baixa amplitude, de curta duração, sucedendo imediatamente o complexo QRS.
\end{tabular}


Nascimento CNG et al. Transplante cardíaco na displasia arritmogênica do ventrículo direito.

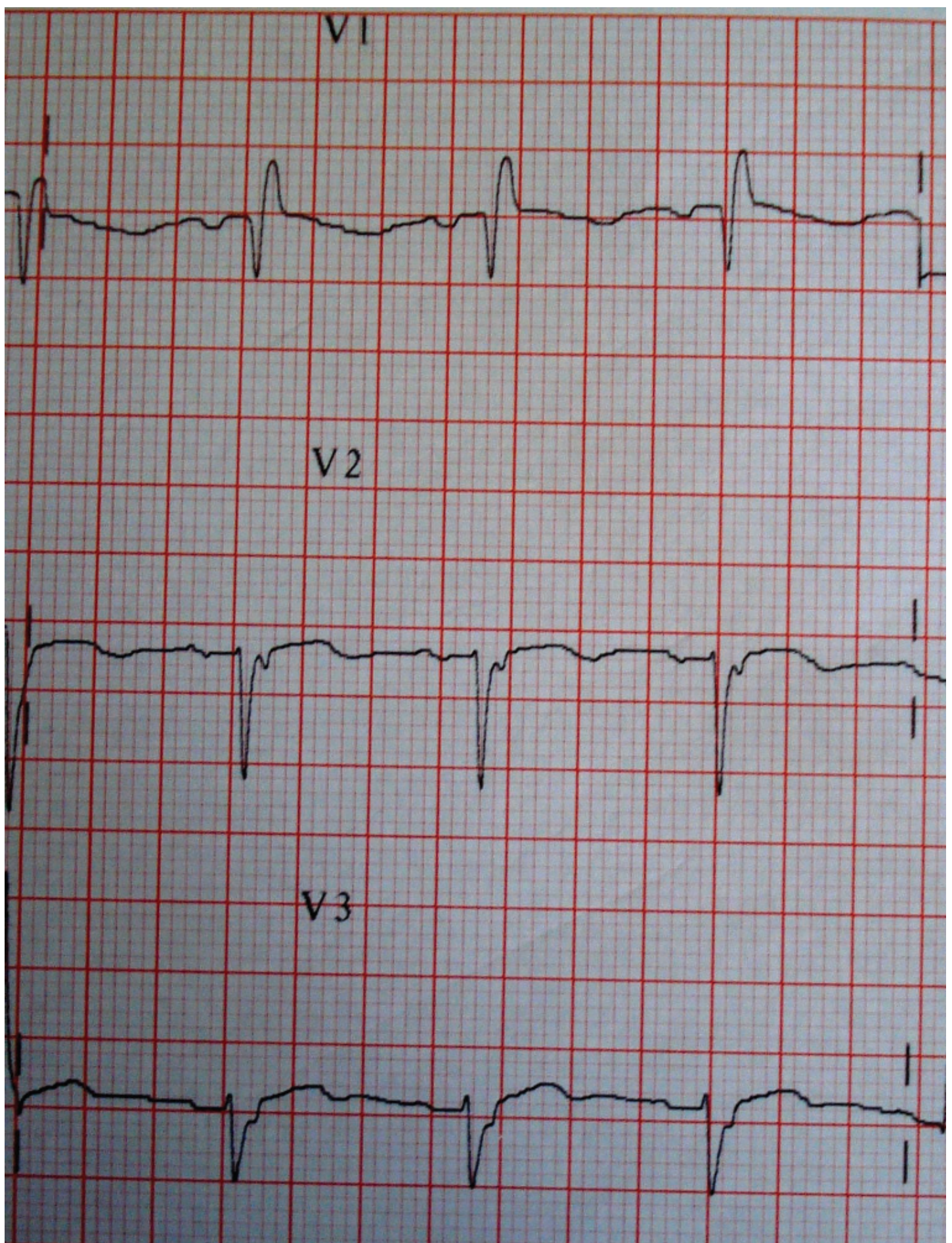

Figura 2. Imagem de eletrocardiograma da pacinte VDP (Caso 1) mostrando derivações V1, V2 e V3. Seta indica onda épsilon nas derivações V2 e V3

Em ecocardiograma (ECO), todos os casos apresentavam dilatação de ambas as câmaras, mais acentuada em ventrículo direito e fração de ejeção diminuída, alterações sugestivas de ICC também encontradas em portadores de DAVD. ${ }^{5}$

O resultado da análise anatomopatológica dos quatro pacientes confirmou o diagnóstico clínico de DAVD, por apresentar substituição do miocárdio por tecidos gorduroso e fibroso com acometimento de grande parte do ventrículo direito, e invasão do septo e ventrículo esquerdo (Figuras 3 e 4). 


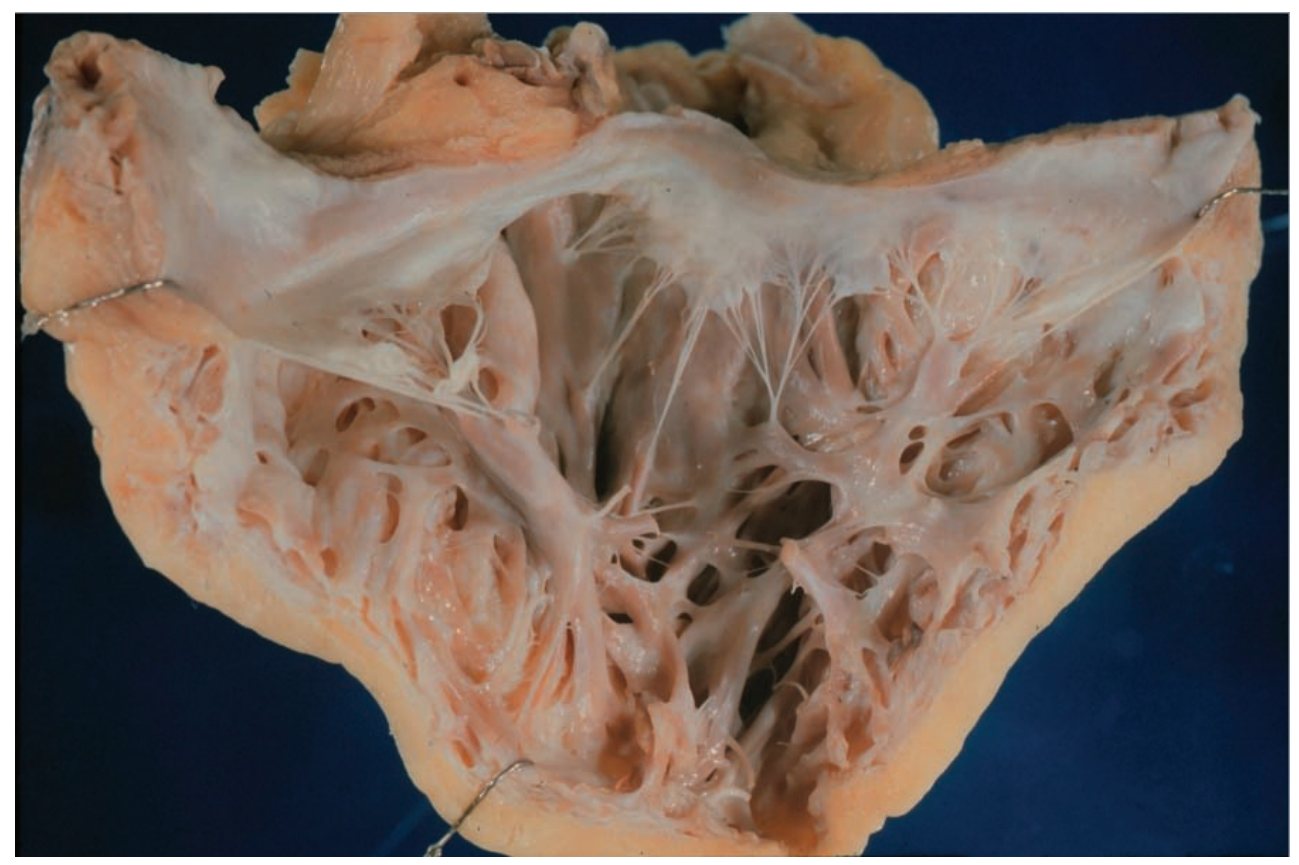

Figura 3. Macroscopia do coração afetado pela DAVD do paciente EDS, evidenciando-se pouquíssimo tecido miocárdico íntegro, e aspecto amarelado, compatível com excesso de tecido fibrogorduroso

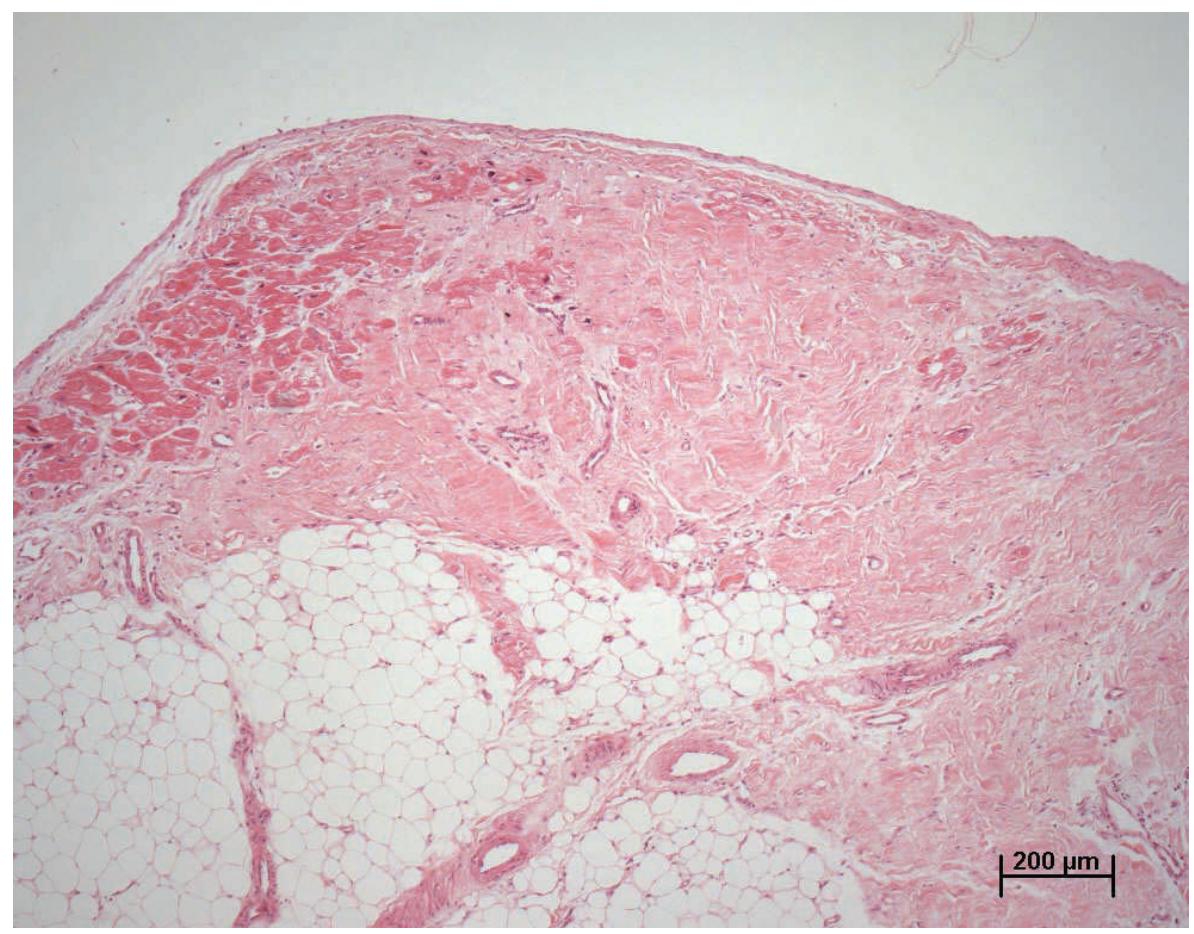

Figura 4. Microscopia do coração apresentado na macroscopia, mostrando substituição do miocárdio por tecido fibrogorduroso

\section{DISCUSSÃO}

A DAVD foi descrita inicialmente em 1977, era antes entendida como parte da miocardiopatia dilatada idiopática. Ainda hoje, seu diagnóstico é confundido com a outra entidade em questão. Na tentativa de solucionar esses problemas, em 1994 foram publicadas as primeiras diretrizes para o seu diagnóstico ${ }^{15}$. 
Nascimento CNG et al. Transplante cardíaco na displasia arritmogênica do ventrículo direito.

Os critérios consistem em dados de história clínica com achados como síncopes, palpitação, sinais de insuficiência de ventrículo direito, por exames não invasivos como eletrocardiograma, ecocardiograma e ressonância nuclear magnética e exames invasivos como angiografia, estudo eletrofisiológico e biópsia endomiocárdica ${ }^{1,4,15}$.

Foi possível, então, estimar a prevalência e a incidência dessa doença, que é respectivamente de 1:1000 e $1: 1250^{15}$ e 6:10000 a 44:10000'.

As disfunções arrítmicas causadas na DAVD têm grande importância quando consideradas as causas de morte súbita, pois na população geral ela é responsável por $10 \%$ dos óbitos; entre os atletas, é a segunda causa de morte súbita, perdendo, somente, para a miocardiopatia hipertrófica ${ }^{19}$.

Comparando-se a evolução da nossa ca- suística com a literatura ${ }^{4,9}$ nota-se que, de fato a progressão da doença foi rápida e grave. $\mathrm{O}$ maior estudo de coorte prospectivo ${ }^{9}$ foi realizado com 130 pacientes com idade média de 31,8 anos $(+/-14,4)$ acompanhados por 8,1 anos (+/-7,8), cuja evolução clínica foi: palpitações $(66,9 \%)$, síncopes $(32,3 \%)$, dor torácica atípica $(26,9 \%)$, dispneia $(10,8 \%)$ e sinais de insuficiência do ventrículo direito (6,2\%). Ainda, 6,2\% eram assintomáticos ${ }^{9}$ (Tabela 3). Apenas $16,5 \%$ dos pacientes faleceram por causa cardíaca, sendo que desses $66,6 \%$ foram por falência cardíaca progressiva e $33,4 \%$ por morte súbita. A baixa mortalidade encontrada, em comparação com outras citações ${ }^{1,15}$ pode ter sido influenciada pela assistência efetiva que esses pacientes receberam. Em apenas 10 pacientes foi necessário o implante de CDI e não foi descrito nenhum caso de transplante.

Tabela 3. Sinais e sintomas mais frequentes apresentados por portadores de DAVD apresentados por Hulot et al. em $2004^{9}$

\begin{tabular}{ll}
\hline Sintomas & Frequência \\
\hline Palpitação & $66,9 \%$ \\
\hline Síncope & $32,3 \%$ \\
\hline Dor torácica atípica & $26,9 \%$ \\
\hline Dispneia & $10,8 \%$ \\
Sinais clínicos de insuficiência do VD & $6,2 \%$ \\
\hline Assintomáticos & $6,2 \%$ \\
\hline Morte súbita & $5,4 \%$ \\
\hline
\end{tabular}

Existem vários fatores de gravidade, que podem explicar a grave evolução que esses pacientes tiveram, como a idade de início precoce (média $18,7 \pm 2,5$ ) e presença de síncopes em três dos casos, que indicam risco de arritmias ${ }^{4,8}$. Ainda, o comprometimento biventricular, que todos os pacientes apresentavam é uma condição rara, que evidentemente demonstra gravidade do quadro.

O tratamento mais utilizado consiste no uso de drogas antiarrítmicas (Sotalol ou Amiodarona) associado ou não a $\beta$-bloqueadores. $O$ implante de um $\mathrm{CDI}^{3,8,17}$ é realizado em pacientes refratários ao tratamento medicamentoso, na prevenção de morte súbita. A ablação por cateter com radiofrequência deve ser considerada quando os dois anteriores não são eficazes, para suprimir os focos de arritmia. A última opção quando há falência ventricular bilateral, com comprometimento hemodinâmico é o tratamento cirúrgico, com ventriculotomia do ventrículo direito ou transplante ${ }^{15,18}$

Devido à baixa frequência de evolução para disfunção biventricular, a experiência na literatura sobre transplantes em DAVD é pequena, sendo que a nossa casuística corresponde a $20 \%$ dos casos já descritos, como mostra a tabela 4.

Tabela 4. Tabela de casos de portadores de DAVD submetidos a transplante cardíaco

\begin{tabular}{llll}
\hline Autor & & Clínico/AP & Local \\
\hline Lu FY et al. $^{11}$ & 2007 & $2 / 9$ & China \\
Greif M et al $^{7}$ & 2008 & $1 / 0$ & Alemanha \\
Korczyk D et al. $^{10}$ & 2007 & $1 / 1$ & Alemanha \\
Peters S $^{16}$ & 2007 & $2 / 2$ & Alemanha \\
Yoda M et al. ${ }^{20}$ & 2005 & $3 / 3$ & Brasil \\
Este estudo & 2008 & $4 / 4$ & \\
\hline Total & & $13 / 19$ & \\
\hline
\end{tabular}


$O$ relato desses quatro casos mostra, portanto, que a Displasia Arritmogênica do Ventrículo Direito pode ser uma doença grave, cursando com arritmias malignas, disfunção ventricular com predomínio do ventrículo direito e morte súbita. O avanço no tratamento clínico e o implante de CDI previnem o aparecimento de morte súbito, aumentando a sobrevida desses pacientes, permitindo, com maior frequência, para falência biventricular. $O$ transplante cardíaco ortotópico deve ser considerado nos casos mais graves e a evolução clínica mostra o caráter curativo do procedimento.

Nascimento CNG, Vilas Boas LB, Napolitano CFC, Coelho GHB, Honorato R, Fiorelli Al. Heart Transplantation in the Arrhythmogenic Right Ventricular Dysplasia. Rev Med (São Paulo). 2009 jan.-mar.;88(1):36-44.

\begin{abstract}
Introduction: The arrhythmogenic right ventricular dysplasia (ARVD) is a myocardial disease, with family origin, were the myocardium is replaced by fibrofatty tissue, with a predominance in the right ventricle. The clinical picture is characterized by the emergence of right ventricular arrhythmias that can lead to sudden death. Objective: This paper aims to present the clinical course of four patients with ARVD, witch were submitted to the orthotopic heart transplant technique bicaval. Patients and Methods: All the four patients had a rapid and severe course, leading to heart failure, with left ventricular involvemtent, and uncontrolled arrhythmias, being indicated the heart transplantation. Results: In all cases the transplant was performed by technical bicaval with the tricuspid valve annuloplasty prophylactic. Only one patient developed hyperacute rejection and infection, leading to death on the $7^{\text {th }}$ day after surgery. The other three cases showed clinical remission of symptoms. The pathological examination of the breasts removed confirmed the presence of the disease. Conclusions: The ARVD is a serious disease that can develop with malignant arrhythmias, ventricular dysfunction with a predominance of the right ventricle and sudden death. The orthotopic heart transplant must be considered in serious cases and shows clinical remission of symptoms.
\end{abstract}

KEY WORDS: Arrhythmogenic right ventricular dysplasia. Heart transplantation. Heart failure.

\title{
REFERÊNCIAS
}

1. Anderson EL. Arrhythmogenic right ventricular dysplasia. Am J Psychiatry. 2008;165(5):649-50.

2. Atik E, Rochitte CE, Avila LF, Kajita LJ, Palhares RB. Prevailing right ventricular myocardiopathy for previous myocarditis or arrhythmogenic dysplasia? Arq Bras Cardiol. 2005;85(1):68-71.

3. Bilchick KC, Judge DP, Calkins H, Marine JE. Use of a coronary sinus lead and biventricular ICD to correct a sensing abnormality in a patient with arrhythmogenic right ventricular dysplasia/cardiomyopathy J Cardiovasc Electrophysiol. 2006;17(3):317-20.

4. Buja G, Estes NA 3rd, Wichter T, Corrado D, Marcus $F$, Thiene $G$. Arrhythmogenic right ventricular cardiomyopathy/dysplasia: risk stratification and therapy Prog Cardiovasc Dis. 2008;50(4):282-93.

5. Elias J, Tonet J, Frank R, Fontaine G. Displasia arritmogênica do ventrículo direito. Arq Brás Cardiol. 1998;70(6):449-56.

6. Fiorelli AI, Stolf NAG, Abreu Filho CAC, Santos RHB, Buco FHA, Fiorelli LR, et al. Prophylactic donor tricuspid annuloplasty in orthotopic bicaval heart transplantation.
Transplant Proc. 2007;39:2527-30.

7. Greif M, Petrakopoulou P, Weiss M, Reithmann C, Reichart B, Nabauer M, et al. Cardiac sarcoidosis concealed by arrhythmogenic right ventricular dysplasia/ cardiomyopathy. Nat Clin Pract Cardiovasc Med. 2008;5(4):231-6.

8. Hodgkinson KA, Parfrey PS, Bassett AS, Kupprion C, Drenckhahn J, Norman MW, et al. The impact of implantable cardioverter-defibrillator therapy on survival in autosomal-dominant arrhythmogenic right ventricular cardiomyopathy (ARVD5). J Am Coll Cardiol. 2005;45(3):409-11.

9. Hulot JS, Jouven X, Empana JP, Frank R, Fontaine G. Natural history and risk stratification of arrhythmogenic right ventricular dysplasia/cardiomyopathy. Circulation 2004;110;1879-84.

10. Korczyk D, Clark P, Crawford D, Maddicks-Law J, Javorsky G, Meyers D. Resolution of protein-losing enteropathy after successful heart transplantation in an adult patient with arrhythmogenic right ventricular cardiomyopathy (ARVC). J Heart Lung Transplant. 2007;26(9):963-4. 
Nascimento CNG et al. Transplante cardíaco na displasia arritmogênica do ventrículo direito.

11. Lu FY, Song LF, Liu L, Zhao H, Wang HY, Li L, et al. Clinicopathologic analysis of dilated heart in cardiac transplant recipients. Zhonghua Bing Li Xue Za Zhi. 2007;36(12):796-800.

12. Moric-Janiszewska E, Markiewicz-Loskot G. Review on the genetics of arrhythmogenic right ventricular dysplasia. Europace. 2007;9(5):259-66.

13. Nijveldt R, Beek AM, Germans T, Bondarenko O, van Rossum AC. Arrhythmogenic right ventricular cardiomyopathy with evidence of biventricular involvement. CMAJ. 2007;176(13):1819-21.

14. Pezawas T, Stix G, Kastner J, Schneider B, Wolzt M, Schmidinger $\mathrm{H}$. Ventricular tachycardia in arrhythmogenic right ventricular dysplasia/cardiomyopathy: Clinical presentation, risk stratification and results of long-term follow-up. Int J Cardiol. 2006;107(3):360-8.

15. Peters $S$. Advances in the diagnostic management of arrhythmogenic right ventricular dysplasiacardiomyopathy. Int J Cardiol. 2006;113(1):4-11.

16. Peters S. Long-term follow-up and risk assessment of arrhythmogenic right ventricular dysplasia/ cardiomyopathy: personal experience from different primary and tertiary centres. J Cardiovasc Med (Hagerstown). 2007;8(7):521-6.

17. Wichter T, Breithardt G. Implantable cardioverterdefibrillator therapy in arrhythmogenic right ventricular cardiomyopathy: a role for genotyping in decisionmaking? J Am Coll Cardiol. 2005;45(3):409-11.

18. Wichter T, Paul TM, Eckardt L, Gerdes P, Kirchhof $\mathrm{P}$, Böcker $\mathrm{D}$, et al. Arrhythmogenic right ventricular cardiomyopathy antiarrhythmic drugs, catheter ablation, or ICD? Herz. 2005;30(2):91-101.

19. Ye D, Edwards WD, Rizkalla W. Sudden unexpected death in a 31-year-old man caused by arrhythmogenic right ventricular cardiomyopathy. Arch Pathol Lab Med. 2005;129:1330-3.

20. Yoda M, Minami K, Fritzsche D, Tendrich G, SchulteEistrup S, Koerfer R. Three cases of orthotopic heart transplantation for arrhythmogenic right ventricular cardiomyopathy. Ann Thorac Surg. 2005;80(6):2358-60. 